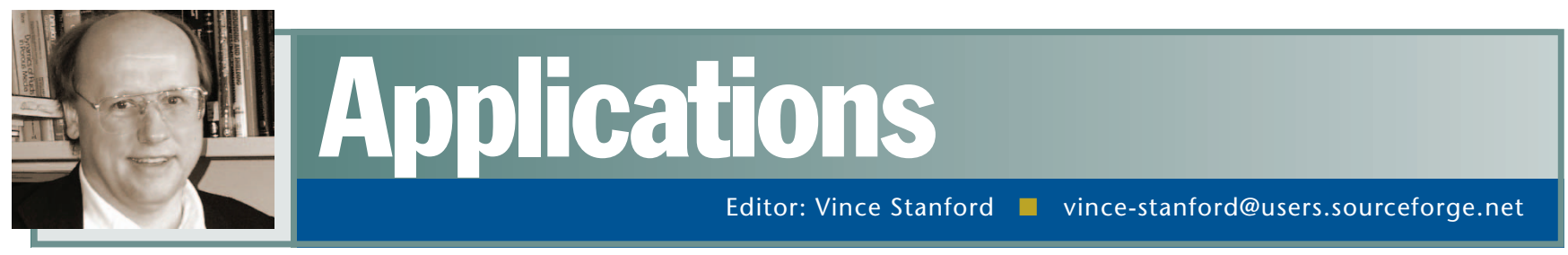

\title{
Wearable Systems in Nursing Home Care: Prototyping Experience
}

Mikael Drugge, Josef Hallberg, Peter Parnes, and Kåre Synnes

\section{EDITOR'S INTRODUCTION}

Productivity in nursing care is a major concern in all residential facilities, as is improving the quality of care. Pervasive computing offers great promise in this area. For example, in the April-June 2003 installment of this column ("Pervasive Computing Goes the Last Hundred Feet with RFID Systems"), we showed how the Vocera mobile voice communication badge can save an hour per day in the busy working lives of nurses by offering voice dialing and voice-over-IP links. This issue's authors discuss requirements analysis, multiphase prototyping, and effective use of commercial off-the-shelf technologies to improve communication and situation awareness in nursing teams. The authors developed a wearable prototype with only a person-month of development time, using existing electronic meeting technologies for its multimodal communication platform. This work suggests interesting research directions, and I hope to provide reports on continuing progress. - Vincent Stanford

edical workers at nursing homes spend much time on communication to get the right information to the right person at the right time. This communication is a prerequisite for proper patient care. Delays cause stress, discomfort, and dissatisfaction among caretakers and patients as well as possible detrimental health consequences for patients. We believe pervasive computing technologies can improve this situation by speeding communication and documenting care more effectively.

In fact, pervasive computing is a promising solution to many problems that medical workers face, and today it's increasingly practical (see "A Pervasive Computing Specialty in Healthcare Emerges" sidebar). Yet actual deployment is still in its infancy. Deploying prototypes that solve specific problems can help medical staff see its benefits. Involv- ing them early in the design process also helps ensure that the right problems are being solved and the solutions will be accepted.

We decided to try rapid prototyping in a real nursing home. We set a tight four-week deadline for ourselves and began work to build a testable prototype with two half-time developers. This gave us one person-month to develop a useful prototype to investigate research questions including:

- What methodologies are useful for prototyping pervasive computing systems?

- How do we engage end users in prototype design and interactions?

- Can we rapidly deploy prototypes built from existing technology in real settings?

- How can we translate conceptual solutions to functional prototypes?
We worked with the Lighthouse, a local nursing home that provides short-term residential care in apartments. Self-sufficient elderly who've been set back by accidents or illnesses can receive the support they need to recuperate. With up to 40 guests, the Lighthouse sees a continuous stream of patients. Although busy, it's small enough for us to easily deploy and study prototypes in real situations.

\section{SCOPING THE PROJECT}

We first had to identify actual problems nurses face in everyday work, determine which could be solved within our research's scope, and identify which would bring the most gain. Although we had reports on typical problems, we decided a field study would give us first-hand experience with their work. This was accomplished by a "quick and dirty" ethnographical study, ${ }^{1}$ where we accompanied a group of four nurses for a day and observed the professional tasks they perform.

During this study, we observed scenarios such as drawing blood samples and administering pain medication. A consistent theme for many tasks was the difficulty in getting necessary patient information at the point of care. The patient charts contain the most important information, but they are only accessible from the nurses' office computers. Nurses typically must walk hundreds of meters and change floors to attend patients in their apartments. 
Going back to the office computers takes time, so the nurses need a system that supports retrieving such information in advance and updating the patient charts upon returning to the office. The nurses currently keep updates in shortterm memory or handwritten notes.

When nurses need more informal information, they must be able to contact the person who previously cared for the patient. Typically, the Lighthouse nurses used mobile phones for this purpose. When the phone calls reached the previous caretaker at all-and often they didn't-they usually interrupted the recipient. Likewise, other people calling the Lighthouse nurses interrupted their patient care, increasing stress and discomfort for both the nurse and patient.

We also found mobile phones lacking multimodal features, supporting only voice, while the situation itself required video to convey certain information. For example, rather than having a patient point out pains directly to a physiotherapist, the nurses must relay this information over the phone, thereby losing the subtle details that body language can reveal.

At the end of the day, we summarized the problems we encountered, both in scenario form and as a list of specific items, including

- communication,

- information dissemination,
- access to patient charts, and

- organizational issues.

A few days later, we went back to the Lighthouse for a meeting with the nurses to validate our findings and ensure that the identified problems were real. This helped us decide which were the most important.

Access to patient charts involves strict privacy and security considerations, and remote accessing requires detailed analysis and evaluation of security infrastructure. So we simulated this information for our prototype. Organizational issues such as staffing, budgets, and work schedules are economic and political issues that we won't discuss further.

We could address the communication and information dissemination issues among the personnel within the limited scope of project. These are closely related, since communication is a way of having the right information for the right person at the right time. We discussed these issues with the nurses and came to a joint conclusion regarding the research prototype's focus. The consensus was that it should support easier communication among the personnel and also function as a documentation tool for informal notes. It should be mobile and allow for access from anywhere in the building, be less intrusive than a mobile phone, and employ a highly streamlined interface to avoid taking focus from the patients.

\section{PAPER PROTOTYPING}

Hardware inventions to embody and run pervasive applications are often wearable or highly portable. Research concepts often rely heavily on unique hardware and require working prototype to test and illustrate the operational concepts. Producing these prototypes can be prohibitively expensive and time consuming. You can simplify hardware prototyping by using modular approaches-for example, the Smart-Its project (www.smart-its.org) operates this way. Yet such prototyping remains focused on hardware technology, which runs the risk of distracting from function and usability.

Traditional HCI researchers have used paper prototyping to good effect. ${ }^{2}$ This simply involves drawing user interface components on paper, making it easy to alter designs and fix flaws early. Not everyone can use design software for prototyping user interfaces, but everyone knows how to draw sketches with a pen. So paper prototyping allows end users to become part of the design process early. Paper prototyping is so artificial that it can remove the focus on technology. Instead, participants can focus on the product's underlying concept and usability. This works, however, because of the fixed and rigid desktop paradigm with its graphical presentation space that can be adequately represented on a sheet of paper.

Paper prototyping in pervasive com-

\section{A PERVASIVE COMPUTING SPECIALTY IN HEALTHCARE EMERGES}

Maintaining social awareness among coworkers is always important for efficient collaboration. It presents special problems in healthcare facilities where the caregivers must go to the patient. Pervasive computing solutions to the productivity and quality issues confronting modern medical practice are increasingly under investigation.

Pervasive healthcare now has its own specialty conference, the first of which will be 29 November through 1 December 2006 (www.pervasivehealth.org). The conference will cover critical topics including design and use of biosensors; continuous versus event-driven patient monitoring; mobile devices for healthcare information storage, update, and transmission; sensor networks; data fusion; pervasive healthcare applications; secu- rity and privacy; systems integration; as well as legal and regulatory issues.

The Aware project at the Centre for Pervasive Healthcare (www.pervasivehealthcare.dk) in Aarhus, Denmark, uses context-aware software to assist caregivers within hospital settings. The Centre's Java ContextAwareness Framework presents a basic technology platform for modeling abstractions including entities, relationships, and context items.

Many laboratories are investigating wearable systems for monitoring patients' health. Amon is one such system, developed at the Wearable Computing Lab at ETH Zürich (www.wearable.ethz.ch), Switzerland. Wearable monitors can simplify the work of the medical personnel as well as improve the quality of healthcare. 
puting applications involves additional challenges. The multimodal interaction in such environments supports more complex scenarios than desktop GUIs accommodate. This freedom can mean the paper itself imposes restrictions on what can be done-for example, not having access to large sheets of paper can prevent participants from envisioning whiteboards, while lacking small sheets can discourage them from thinking of handheld devices.

\section{PAPER, PEN, AND PLASTIC}

Following our requirements study with the nurses, we arranged a meeting to try our technique. We informed them that we needed their input and feedback to design a prototype that would be useful to them and that we would employ paper prototyping. We prepared scripted scenarios from the data we'd collected on typical nursing tasks, such as visiting and examining patients, informing physiotherapists, taking blood samples, and making rounds with physicians. We let them role-play the scenarios and discuss various solutions to problems they encountered. One of the nurses played herself in work situations, while two others played patient roles. Each patient player received a brief description of the scenario.

The nurse used a notepad representing a mobile device, which could be conveniently worn in her belt. We briefly explained the prototype's general functionality for contacting someone like the previous caretaker, capturing video using a folded paper "camera," taking notes by voice or text, accessing a patient's medical history, and so on. We took care not to provide instructions regarding how to use the prototype. Instead, we observed how the nurses intuitively used it and let those observations guide further design.

In addition to the scenario, we had text cards representing typical phone calls. We emulated a context-aware service that could determine whether the nurse was currently occupied and could then choose between presenting phone calls directly or taking a message. For calls that weren't urgent, we displayed a simulated text message on a transparent piece of plastic. We then either set it in front of the nurse to view in private or placed it on the device the nurse carried. Presenting these messages randomly during the role play allowed the nurses to decide what visualization form they preferred.

After completing the scenario, we talked with the nurses about their experience. They appreciated being able to avoid interruptions from nonacute calls through simple mechanisms such as screening incoming messages. They liked having text messages presented because it let them read without interrupting what they were doing. They found it useful to have a patient's information available in advance or upon contact because it let them better prepare for encounters with special patients. For example, if a patient posed difficulties in taking blood samples, the nurses could add extra test tubes to their medical kits. The nurses also liked having the live video to directly show, for example, a patient's shoulder fracture. They thought this would save them time that they could spend on more important tasks. In general, the paper prototyping session made the prototype's benefits clear without having to deploy fully functional hardware and software.

\section{PAPER PROTOTYPING BENEFITS}

The benefits of early paper prototyping over an online, functional softwarehardware prototype became clear at the end of the meeting. We brought some hardware just to show the nurses what is available with today's technology. Immediately, we noticed their focus shifting away from usefulness to technical details regarding the software and hardware. They questioned font sizes ("too small for me to read"), commented on video quality ("better than the one we saw on another project"), and expressed astonishment over features ("so I could even write my emails on this"). As their focus scattered, they often addressed details irrelevant to the tasks they would need to perform.

Importantly, while interacting with the computer, we noticed the nurses started to think in terms of traditional user interface widgets, such as buttons, menus, and keyboards, and to restrict themselves to the kind of interactions typical of desktop PC applications. They also appeared more dejected and hesitant to suggest improvements, and they expressed slightly negative comments and questions such as, "We'll need to take courses to understand this. You'll arrange that for us, right?" In general, the nurses shifted their entire focus, assuming now that only minor changes in technical details were possible. Clearly, such restrictions in the design space should not dominate the early stages of prototyping.

We concluded that paper prototyping in the initial development stages makes participants focus more on a product's concept and actual usefulness rather than letting technology constrain their thinking and dictate what is allowed. Furthermore, unlike technology, paper does not restrict the design space, allowing participants to think beyond the inherent limitations of current software and hardware. We see the same benefits for pervasive computing research that it exhibits for traditional HCI in desktop computing. Taking merely one week in preparation, execution, and analysis, we deem this time well spent.

\section{MOVING TO MULTIMODAL DEVICES}

With the newfound design considerations from the paper prototyping session in mind, we went on to see what research technologies could help in realizing a multimodal prototype. Our research group's background is based in real-time audio and video communication over the Internet, and we have extensive experience in desktop e-meeting tools. We've also ventured into the pervasive computing field, investigating mobile and ubiquitous applications of the technology. Beyond technical barriers, we've found that the end users' con- 
cerns and preconceptions often determine whether new systems are adopted.

For this application, the prototype had to be mobile and nonintrusive, which fits well within the field of wearable computing. We also saw healthcare applications requiring special considerations, as illustrated in one nurse's opinion: "It was not to sit in front of computers I chose this profession." The prototype had to avoid the negative emotions that time-stealing and crashprone desktop computers currently cause. An important way to hide technology and streamline the interaction is to use context and situation awareness, automating the information presented to the nurses according to the current activity and workload.

Some of these concerns are major research problems in themselves, which meant that our online prototype couldn't realize all concepts within a reasonable time frame. However, by employing a Wizard of $\mathrm{Oz}$ approach to the user interface, ${ }^{3}$ we could still demonstrate the functionality and get the nurses' opinions. Knowing how, or whether, the nurses used certain functions let us select what areas to put the most effort on in future prototypes.

\section{WEARABLE PROTOTYPE}

The next step was to build an online prototype with live software and hardware. We wanted the prototype ready within a week from the paper prototyping session. This gave us no time to build customized hardware, meaning we had to assemble our prototype from off-the-shelf products.

As a wearable computer, we chose a Sony Vaio U70P, a notebook computer with a $1-\mathrm{GHz}$ Pentium mobile processor and 512 Mbytes of memory. With dimensions of $16.7 \times 11 \times 2.8 \mathrm{~cm}$ and weighing $550 \mathrm{~g}$, it can be worn easily by strapping it to a belt, which the nurses deemed suitable during our last meeting.

Because the nurses liked viewing information in private, we added a head-mounted display as an alternative to looking at the U70P. We chose the

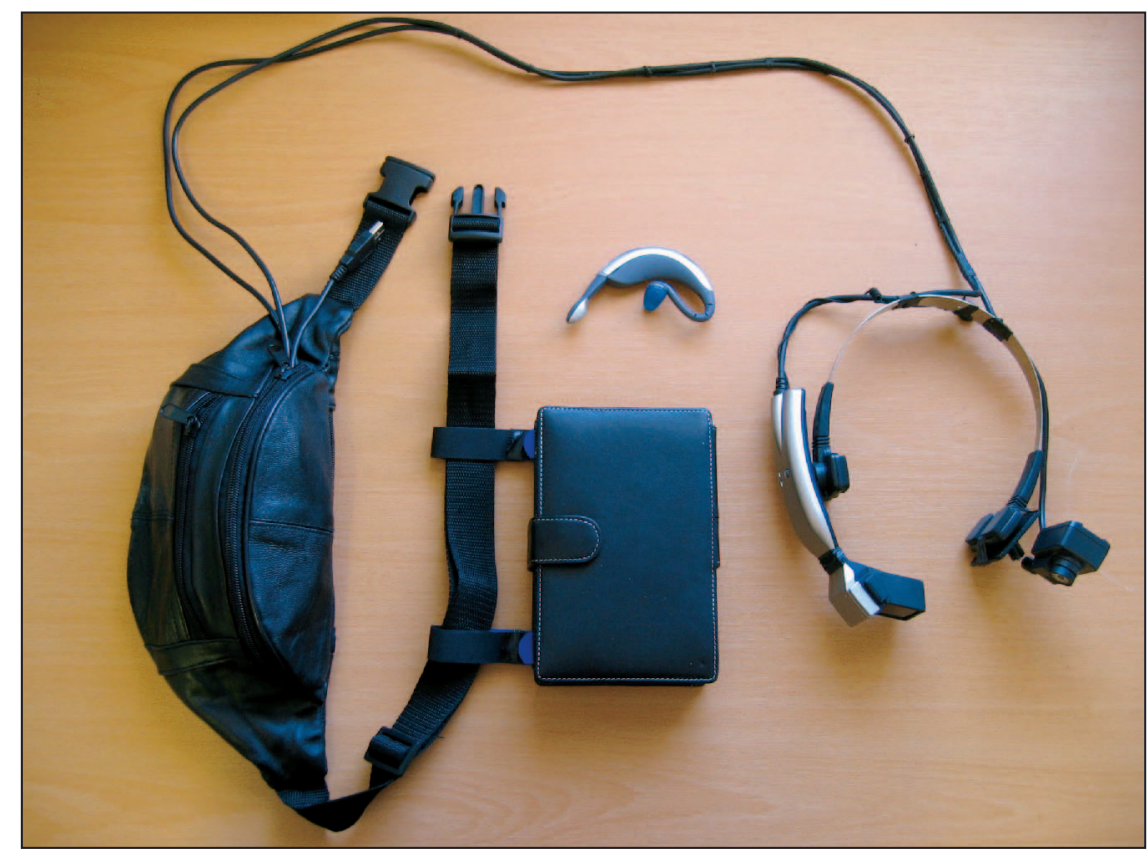

Figure 1. Wearable computer outfit: battery pack and VGA converter for the headmounted display (left), U70P computer (lower center), Bluetooth headset (upper center), and M2 head-mounted display (right).

semitransparent monocular M2 Personal Viewer with full-color graphics in $800 \times 600$ resolution, even though it requires a half-kilogram battery. Since the prototype demonstrates concepts rather than a final product, we deemed the quality of graphics to be more important than the additional weight at this stage. Figure 1 shows the wearable computer and display.

\section{COMMUNICATION APPLICATION}

We chose Marratech (www.marratech. com) e-meeting application software because it fits the communication needs of the envisioned prototype. Marratech is a commercial product based on earlier research in our group. ${ }^{4}$ It allows for audio and video group communication, together with text chat and a shared whiteboard. Connecting the wearable computer to an e-meeting over a wireless network lets the nurse instantly contact other participants and become aware of their locations. The application also lets a nurse make phone calls, which can become part of the e-meeting. This allows persons not yet using the software to be included, easing its deployment.

Figure 2 shows the wearable computer running the Marratech Pro application, with video streams provided by Web cameras. The nurse can use the camera to convey live video to physiotherapists or others to aid diagnoses.

\section{WIZARD OF OZ TESTING}

We wanted to show a simple and automatic system to the nurses, so we decided on a Wizard of Oz experiment to simulate the context-aware and situationaware system components. The wizard retrieved information, processed interrupting phone calls, simplified communication with others, and minimized the interaction needed with the wearable computer.

Each morning the nurses make several calls for information regarding their patients that day. In our prototype, the wizard collects this information and displays it in the shared whiteboard, thus shortening the time nurses need to set their daily schedule. With access to patients' charts, historical notes, and other nurses' locations and 


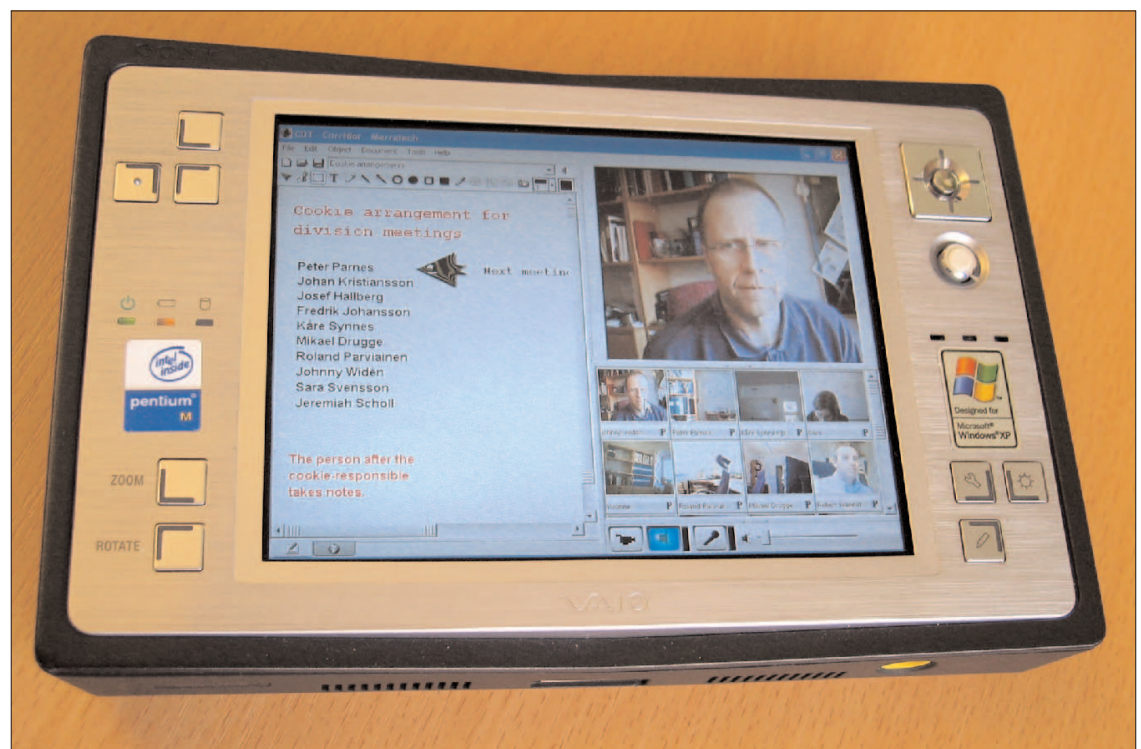

Figure 2. The Sony Vaio U70P running the Marratech Pro e-meeting application.

schedules, the wizard can post appropriate information throughout the day.

If a nurse is attending to a patient or is otherwise busy, the wizard intercepts all phone calls. It sends only urgent calls directly to the nurse and either records

the rest or posts them as a chat room message to read when there is time. This substantially reduces interruptions during patient encounters. When nurses wish to reach someone, the wizard can invite that person into the e-meeting

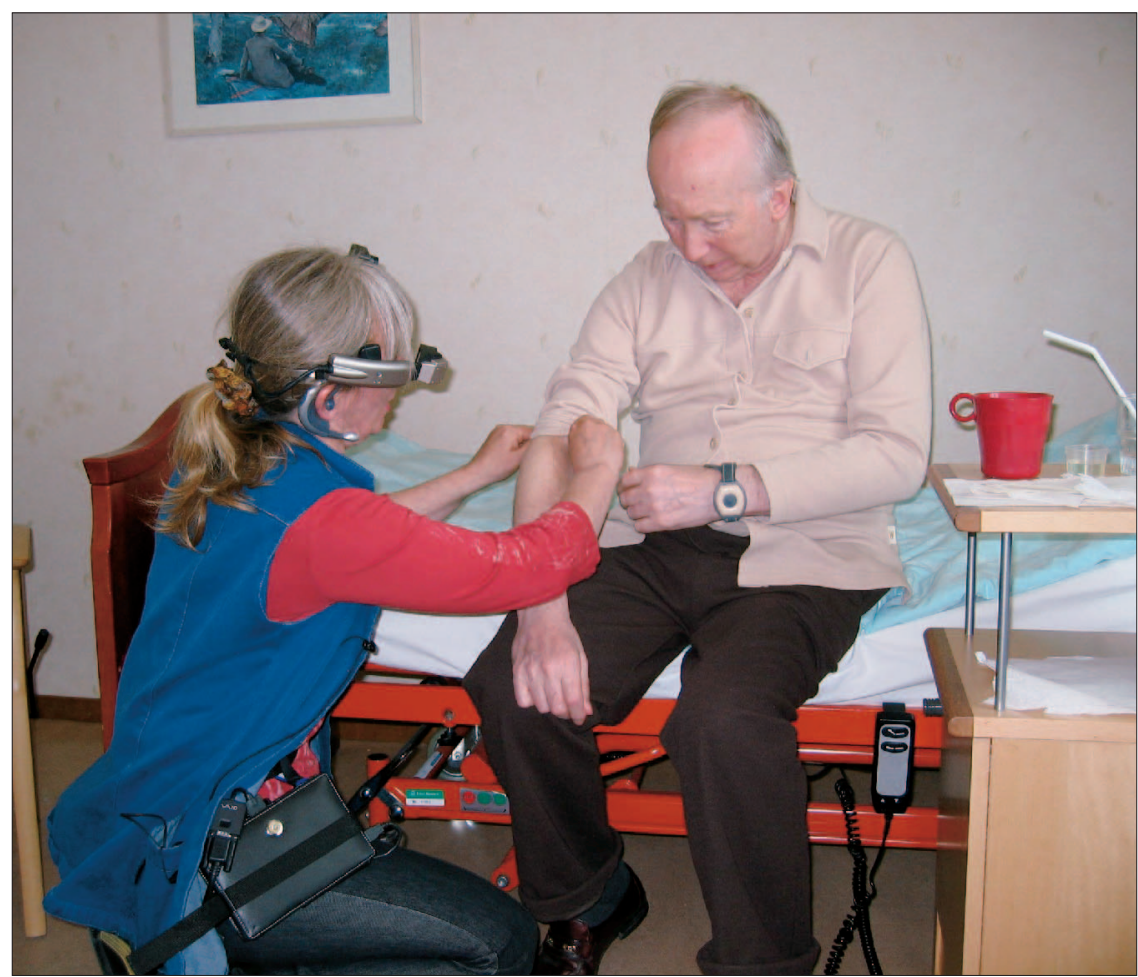

Figure 3. A nurse wearing a computer and head-mounted display while attending a patient. session, thus allowing richer communication than normal phones.

To further simplify the nurses' work, we let the wizard act as a speech recognizer. Nurses can take informal notes about certain patients for insertion into their chart. They can also request information to be read out loud, which further simplifies the interaction with the wearable computer. In addition, the nurses can use voice to insert new tasks into their schedules.

Most of the wizard's functions can be realized with today's technology, such as RFID tags, sensors, and speech recognition capabilities.

\section{FEEDBACK FROM THE NURSES}

We brought our wearable prototype to the Lighthouse to test it for a day, starting with audio-only communication. We equipped one nurse with the wearable computer and a Bluetooth headset, while another nurse in another room used a laptop. We connected both devices to the same e-meeting, which effectively mimicked mobile phones but reduced interruptions and offered higher audio quality. We also allowed review of a fictitious patient history and chart to demonstrate the capability.

Next, we introduced video communication. As one e-meeting participant walked the corridors, the nurses expressed their fascination with instantly seeing where their colleagues was. This increased group awareness seemed beneficial, especially for finding the right person at the right time. Initially, the nurse sent video via a handheld camera and used the notebook's display to view other participants. Because these tasks encumber the nurse's hands, we also let them test the head-mounted display and camera. After a brief time, about 15 minutes, they accustomed themselves to the novel display and learned to focus on the display or the real world as needed. Soon the nurses could easily perform routine patient examinations while conveying video to other medical workers. This aided patient diagnosis and treatment deliberations. Figure 3 shows a patient 
encounter. Although noting the added weight, the nurses remained focused on the design concept being demonstrated and its benefits.

Thus, on the basis of results from paper prototyping and a wearable prototype built from off-the-shelf components, we successfully demonstrated the envisioned benefits and gained user acceptance for an assistive device in this environment. By starting with the basic functions and gradually adding more, we avoided intimidating the users with the amount of hardware and cables.

bout a month after the online study, we revisited the Lighthouse to discuss the prototyping in hindsight. Despite the time that had passed, the nurses still deemed the prototype useful and appropriate. We see this as confirming that the process yielded usable results and that ethnographical studies and paper prototyping can be effective in pervasive computing research.

Ethnographical study provides valuable first-hand insight on how work is performed and gains the confidence of the user community. Paper prototyping offers a cheap and easy way to get quick feedback on what constitutes a good or bad solution. Furthermore, people not in the pervasive computing research community still consider much of what is possible with today's technology as science fiction. We found it difficult to get those people to envision what's possible, and we had to find ways of freeing them from traditional PC interface ideas, while not imposing our ideas on how things should be done. Paper prototyping can aid this to some degree, and joint discussions of paper results encourage fuller exploration of the design space. It also moves the participants' focus from technology to usability and function.

One major challenge we found concerning paper prototyping is to communicate what is realizable without constraining participants from exploring the whole design space spectrum. We think the best solution is to intro- duce concepts in a brainstorming session before the paper prototyping. You can add the wildest ideas to the hardware research agenda and use ideas that can be realized with current hardware technology in the prototype. This should allow for more freedom of thought in the subsequent paper-prototyping stage while constraining the overall process to prototypes possible given the available time, budget, and technology.

Realizing the paper prototype in functional hardware reveals differences between reality and visions that can require compromises. The Wizard of $\mathrm{Oz}$ approach allows for emulation of functionality not immediately realizable. However, if the prototype is meant to be deployed for longer term studies, the researcher should be sure this functionality is realizable within the envelope of current technology.

Finally, rapid prototyping with enduser involvement from the start has a value in itself. The nurses we worked with spontaneously expressed enthusiasm for our project due to its fast pace. They felt that something was happening and that their input was valued. As opposed to other projects where meetings are half a year apart, rapid prototyping let them see progress from week to week. P

\section{ACKNOWLEDGMENTS}

We wish to acknowledge funding by the Centre for Distance-spanning Healthcare at Luleå University of Technology. We also express our thanks to the nurses and medical workers at the Lighthouse (in Swedish, "Fyrens korttidsboende") in Luleå, Sweden, for their participation. We also thank the reviewers for valuable guidance.

\section{REFERENCES}

1. J. Hughes et al., "The Role of Ethnography in Interactive Systems Design," Interactions, vol. 2 , no. 2,1995 , pp. 56-65

2. M. Rettig, "Prototyping for Tiny Fingers," Comm. ACM, vol. 37, no. 4, 1994, pp. 21-27.

3. N. Dahlbäck, A. Jönsson, and L. Ahren- berg, "Wizard of Oz Studies: Why and How," Proc. 1st Int'l Conf. Intelligent User Interfaces, ACM Press, 1993, pp. 193-200.

4. P. Parnes, K. Synnes, and D. Schefström, "mStar: Enabling Collaborative Applications on the Internet," IEEE Internet Computing, vol. 4, no. 5, 2000, pp. 32-39.

Mikael Drugge is a PhD student in the Media Technology research group at Luleå University of Technology. Contact him at Luleå Univ. of Technology, Dept. of

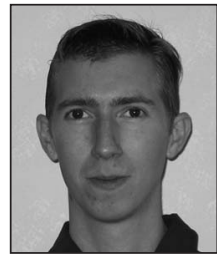
Computer Science and Electrical Eng., SE-971 87 Luleå, Sweden; mikael.drugge@ltu.se.

Josef Hallberg is a PhD student in the Media Technology research group at Luleå University of Technology. Contact him at Luleå Univ. of Technology, Dept. of

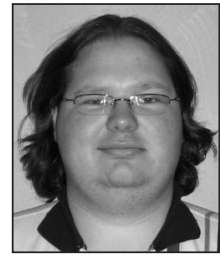
Computer Science and Electrical Eng., SE-971 87 Luleå, Sweden; josef.hallberg@ltu.se.

Peter Parnes is an associate professor and the research leader for the Media Technology research group at Luleå University of Technology. Contact him at Luleå

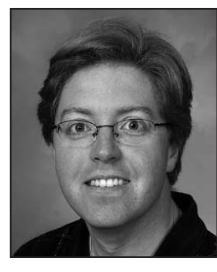
Univ. of Technology, Dept. of Computer Science and Electrical Eng., SE-971 87 Luleå, Sweden; peter.parnes@ltu.se.

Kåre Synnes is an assistant professor and acting head of the Information and Communication Technology division at Luleå University of Technology. Contact him at

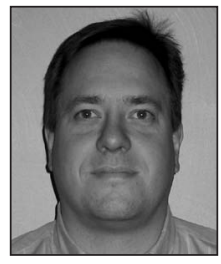
Luleå Univ. of Technology, Dept. of Computer Science and Electrical Eng., SE-971 87 Luleå, Sweden; kare.synnes@ltu.se. 